\title{
ОЦЕНКА КОРРЕЛЯЦИОННОЙ ДЛИНЫ В ВИБРОННОМ СЕГНЕТОЭЛЕКТРИКЕ
}

\author{
(Представил В. Хижняков)
}

Рассмотрены свойства вибронного сегнетоэлектрика, связанные со статическими неэднородностями фононного параметра порядка. Оценены коэффициент корреляционной энергии, корреляционная длина (оказывается пропорциональной квадрату энергии ведущего переход межзонного вибронного взаимодействия) и число Гинзбурга-Леванюка для $\mathrm{BaTiO}_{3}$ и узкощельных еистем типа $\mathrm{A}_{\mathrm{Iv}} \mathrm{Bv}$.

Одним из наиболее актуальных направлений в теории структурных фазовых переходов в настоящее время является изучение области критических флуктуаций, где обычная теория Ландау, построенная без их учета, становится неприменимой. Важной задачей является, естественно, определение границ этой области, в частности, корреляционной длины, связанной с пространственно-неоднородными флуктуациями параметра порядка. Принципиальные стороны этой проблемы рассматривались впервые В. Л. Гинзбургом и А. П. Леванюком $\left[{ }^{1,2}\right]$. Для сегнетоэлектриков с переходом типа смещения оценки корреляционной длины выполнены в $\left[{ }^{1-3}\right]$ с использованием эффективных дипольных сил. Детальная микроскопическая модель ведущего фазовый переход взаимодействия при этом не использовалась.

Разработанная относительно недавно вибронная теория сегнетоэлектричества $\left[{ }^{4-8}\right]$ усматривает микроскопическую причину возникновения переходов типа смещения в электрон-фононном межзонном взаимодействии. На основании этой теории до сих пор исследовались системы двух типов: а) сегнетоэлектрики-полупроводники типа $\mathrm{A}_{\mathrm{IV}} \mathrm{B}_{\mathrm{vI}}{ }^{*}$, б) широкощельные сегнетоэлектрики-диэлектрики типа $\mathrm{BaTiO}_{3}$. В первом случае активное вибронное взаимодействие, обусловливая нестабильность активной в переходе фононной моды, может служить стабилизации высокосимметричной фазы с ростом температуры за счет вибронно индуцированного ангармонизма $\left[{ }^{11-13}\right]$, причем следует учитывать и изменения заселенностей зон $\left[{ }^{14}\right]$. Во втором случае межзонное электрон-фононное взаимодействие обусловливает нестабильность актуальной моды (мнимость ее исходной частоты), а стабилизирующие температурные зависимости вносятся фононным ангармонизмом, доминирующим над вибронным $\left[{ }^{15}\right]$. В вибронной теории о динамических флуктуациях критической ветви $(c \mathrm{q} \neq 0)$ обычно говорят в целях обозначения факторов (упомянутой природы), стабилизирующих высокосимметричную фазу и вносящих в теорию определяющие температурные зависимости через фононные числа заполнения. Учет межзонного электрон-фононного взаимодействия в этих целях должен выходить за рамки приближения хаотических фаз $[4,11,16,17]$. Соответствующие поправки существенны для узкощельных систем, как уже отмечалось.

* Относительно микроскопической теории систем $\mathrm{A}_{\mathrm{Iv}} \mathrm{B}$ І см. также $\left[{ }^{9,10}\right]$. 
В настоящем сообщении будут рассмотрены простран̈ственно-нёоднородные флуктуации фононного параметра порядка $y(\mathbf{r})$ в вибронном сегнетоэлектрике. Основная задача при этом заключается в получении выражения для коэффициента $c$ при корреляционной энергии в разложении плотности свободной энергии

$$
f=a k_{B}\left(T-T_{C}\right) y^{2}+\frac{1}{2} b y^{4}+c|\operatorname{grad} y|^{2}
$$

qерез микроскопические характеристики кристалла.

Область применимости обычной теории Ландау определяется малостью числа Гинзбурга-Леванюка $\left[{ }^{18}\right]$

$$
G_{i}=\frac{k_{B} b^{2} T_{C}}{a c^{3}} \ll 1
$$

где $T_{C}-$ температура Кюри, $k_{B}-$ постоянная Больцмана. Корреляционная длина для низкотемпературной фазы определяется соотношением

$$
\xi^{2}=\frac{c}{2 k_{B} a}\left(T_{C}-T\right)^{-1}=\xi_{0}^{2} T_{C}\left(T_{C}-T\right)^{-1} .
$$

Переходя в (1) к Фурье-образам можно первый и третий член в (1) объединить в следующий квадратичный по фононным переменным член в свободной энергии

$$
\Phi=N_{0} v \sum_{\mathbf{q}}\left(a k_{B}\left(T-T_{C}\right)+c \mathbf{q}^{2}\right) y_{\mathbf{q}} y_{-\mathbf{q}}=\frac{1}{2} \sum_{\mathbf{q}} M \Omega^{2}(\mathbf{q}) y_{\mathbf{q}} y_{-\mathbf{q}} .
$$

Отсюда видна связь коэффициента при $\mathbf{q}^{2}$ в квадрате частоты мягкой ветви колебаний с коэффициентом $c$ корреляционной энергии в (1). Через $N_{0}$ обозначено число элементарных ячеек, $v$ - объем ячейки. Мы будем основываться на расчете величины $M \Omega^{2}(q)$ в форме (4) по вибронной теории. Гамильтониан вибронного сегнетоэлектрика возьмем в обычном виде $\left[{ }^{4}\right]$

$$
\begin{gathered}
H=\sum_{\sigma, \mathbf{k}} \varepsilon_{\sigma}(k) a_{\sigma \mathrm{k}}^{+} a_{\sigma \mathrm{k}}+\frac{1}{2} \sum_{\mathrm{q}}\left(M \omega_{\mathrm{q}}^{2} y_{\mathrm{q}} y_{-\mathrm{q}}+\frac{1}{M} P_{\mathrm{q}} P_{-\mathrm{q}}\right)+ \\
+\frac{1}{N_{0}} \sum_{\sigma, \sigma^{\prime}} \sum_{\mathbf{k}, \mathrm{q}} V_{\sigma \sigma^{\prime}}(\mathrm{q}) a_{\sigma \mathrm{k}}^{+} a_{\sigma^{\prime} \mathrm{k}-\mathrm{q}} y_{\mathrm{q}}+\frac{1}{4 ! N_{0}} \sum_{\{\mathrm{q}\}} B\{\mathrm{q}\} y_{\mathrm{q}_{1}} y_{\mathrm{q}_{2}} y_{\mathrm{q}_{3}} y_{\mathrm{q}_{\mathrm{s}}} \delta\{\mathrm{q}\} .
\end{gathered}
$$

В качестве затравочного электронного спектра $\varepsilon_{\sigma}(\mathbf{k})$ выберем валентную зону и зону проводимости с изотропными квазисвободными законами дисперсии, разделенные щелью $\Delta$ при $\mathrm{k}=0$. Динамические переменные мягкой моды обозначены через $y_{q}$ и $P_{q}$, третий член в (5) описывает ведущее вибронное взаимодействие, $B\left(\mathbf{q}, \mathbf{q}^{\prime}\right)=B(\mathbf{q},-\mathbf{q}$, $\left.\mathbf{q}^{\prime},-\mathbf{q}^{\prime}\right)$ - константа собственного ангармонизма критической ветви, функция $\delta\{q\}$ учитывает закон сохранения фононных квазинмпульсов. Дисперсия затравочных размягчающихся колебаний имеет вид

$$
\omega_{\mathrm{q}}^{2}=\omega_{0}^{2}-\alpha \mathbf{q}_{\mathrm{q}}^{2} .
$$

В приближении хаотических фаз для вибронного взаимодействия и псевдогармоническом по фононному ангармонизму перенормированная частота критической фононной ветви при полностью заполненной валентной зоне и пустой зоне проводимости дается соотношением $\left[{ }^{17}\right]$ 


$$
\begin{aligned}
& M \Omega^{2}(\mathbf{q})=M \omega_{\mathbf{q}}^{2}-\frac{1}{N_{0}} V^{2}(\mathbf{q}) \sum_{\mathbf{k}}\left\{\left[\varepsilon_{2}(\mathbf{k}+\mathbf{q})-\varepsilon_{1}(\mathbf{k})\right]^{-1}+\right. \\
& \left.+\left[\varepsilon_{2}(\mathbf{k})-\varepsilon_{1}(\mathbf{k}+\mathbf{q})\right]^{-1}\right\}+\frac{\hbar}{2 N_{0}} \sum_{\mathbf{q}^{\prime}} \frac{B\left(\mathbf{q}, \mathbf{q}^{\prime}\right)}{M \omega_{\mathbf{q}^{\prime}}} \operatorname{coth} \frac{\hbar \omega_{\mathbf{q}^{\prime}}}{2 k_{B} T} .
\end{aligned}
$$
имеем

Разлагая (7) в ряд по q до квадратичных членов включительно,

$$
M \Omega^{2}(\mathbf{q})=\overline{M \omega_{0}^{2}}+2 \bar{a} k_{B} T+2 \bar{c} \mathbf{q}^{2}
$$

причем

$$
\begin{gathered}
M \overline{\omega_{0}^{2}}=M \omega_{0}^{2}-\frac{2}{N_{0}} V^{2}(0) \sum_{\mathbf{k}}\left[\varepsilon_{2}(\mathbf{k})-\varepsilon_{1}(\mathbf{k})\right]^{-1}, \\
M \bar{\omega}_{0}^{2}+2 \bar{a} k_{B} T=2 \bar{a} k_{B}\left(T-T_{C}\right)
\end{gathered}
$$

и мы воспользовались аппроксимацией

$$
\frac{\hbar}{2 N_{0}} \sum_{\mathbf{q}} \frac{B(0, \mathbf{q})}{M \omega_{\mathrm{q}}} \operatorname{coth} \frac{\hbar \omega_{\mathrm{q}}}{2 k_{B} T} \approx \frac{k_{B} T}{N_{0}} \sum_{\mathbf{q}} \frac{B(0, \mathbf{q})}{M \omega_{\mathbf{q}}^{2}}=2 \bar{a} k_{B} T .
$$

Очевидно, что

$$
a=\bar{a}\left(N_{0} v\right)^{-1}, \quad c=\bar{c}\left(N_{0} v\right)^{-1} .
$$

Согласно (7) вклады в $c$ имеют следующее происхождение.

1. Вклад от дисперсии фононного ангармонизма. Используя аналогично $\left[{ }^{19}\right]$ аппроксимацию

$$
B\left(\mathbf{q}, \mathbf{q}^{\prime}\right)=B \omega_{\mathbf{q}}^{2} \omega_{\mathbf{q}^{\prime}}^{2} .
$$

где $\omega_{q}$ дано (6), получаем

$$
\bar{c}_{1}=-\frac{\bar{a} \alpha}{\omega_{0}^{2}} k_{B} T .
$$

Неизвестную постоянную $B$ можно оценить на основании (11) из соотношения $2 \bar{a} \approx B \omega_{0}^{2} M^{-1}$, поскольку дисперсия затравочной частоты $(\alpha)$ мала.

2. Вклад от дисперсии константы межзонного вибронного взаимодействия $V(\mathbf{q})$. На основании результатов $\left[{ }^{20}\right]$ следует оценка для малых q

$$
V^{2}(\mathbf{q}) \simeq V^{2}(0)\left(1-d^{2} \mathbf{q}^{2}\right),
$$

где $d-$ параметр решетки. Соответственно

$$
\bar{c}_{2}=\frac{1}{N_{0}} V^{2}(0) d^{2} \sum_{\mathbf{k}}\left[\varepsilon_{2}(\mathbf{k})-\varepsilon_{1}(\mathbf{k})\right]^{-1} .
$$

3. Вклад от дисперсий электронных зон, дыя которых принимается $\varepsilon_{1}(\mathbf{k})=-\hbar^{2} \mathbf{k}^{2} / 2 m_{p}, \varepsilon_{2}(\mathbf{k})=\Delta+\hbar^{2} \mathbf{k}^{2} / 2 m_{n}$. Линейные по $\mathbf{k}$ члены при суммировании по $\mathrm{k}$ выпадают, так что

$$
\bar{c}_{3}=\frac{\hbar V^{2}(0)}{6 \mu N_{0}} \sum_{\mathbf{k}} \frac{\varepsilon_{2}(\mathrm{k})-\varepsilon_{1}(\mathrm{k})+2 \Delta-2\left[\varepsilon_{2}(\mathrm{k})-\varepsilon_{1}(\mathrm{k})-\Delta\right] \gamma^{2}}{\left[\varepsilon_{2}(\mathrm{k})-\varepsilon_{1}(\mathrm{k})\right]^{3}},
$$

где

$$
\mu=2 m_{n} m_{p} /\left(m_{n}+m_{p}\right), \quad \gamma=\left(m_{n}-m_{p}\right) /\left(m_{n}+m_{p}\right) \text {. }
$$


4. Виклад от затравочной диспіерсий мягкой моды (6)

$$
\bar{c}_{4}=-\frac{1}{2} \alpha M
$$

Переходя далее от суммирования по $\mathbf{k}$ к интегрированию по энергии и собирая вклады $\bar{c}_{i}(i=1, \ldots, 4)$, получаем $(V=V(0))$

$$
\begin{gathered}
\bar{c}=\frac{\hbar V^{2}}{2 \mu E_{0}^{3 / 2}} \int_{0}^{E_{0}} \frac{E+3 \Delta-2 E \gamma^{2}}{(E+\Delta)^{3}} \sqrt{E} d E+ \\
+\frac{3 V^{2} d^{2}}{E_{0}^{3 / 2}} \int_{0}^{E_{0}} \frac{\sqrt{E} d E}{E+\Delta}-\frac{\alpha}{2 \omega_{0}^{2}}\left(M \omega_{0}^{2}-2 \bar{a} k_{B} T\right),
\end{gathered}
$$

причем $E_{0}=\hbar \mathbf{k}_{0}^{2} / \mu-$ суммарная ширнна разрешенных $3 о н, k_{0}^{3}=$ $=6 \pi^{2} / v$.

Последними двумя членами в (19) можно в меру малости затравочной дисперсии активного оптического колебания пренебречь. Выполняя в (19) интегрирование, находим

$$
\begin{gathered}
\bar{c}=\frac{V^{2} d^{2}}{4 \delta}\left[\frac{3\left(1-\gamma^{2}\right)}{\sqrt{E_{0} \Delta}} \arctan \sqrt{\frac{E_{0}}{\Delta}}-\frac{E_{0}+3 \Delta-\left(5 E_{0}+3 \Delta\right) \gamma^{2}}{\left(E_{0}+\Delta\right)^{2}}\right]+ \\
+\frac{6 V^{2} d^{2}}{E_{0}}\left[1-\sqrt{\frac{\Delta}{E_{0}}} \arctan \sqrt{\frac{E_{0}}{\Delta}}\right]
\end{gathered}
$$

где обозначено $\delta=\left(6 \pi^{2}\right)^{2 / 3}$.

Для систем с узкой запретной щелью и широкими разрешенными зонами (сегнетоэлектрики-полупроводники) появляется малый параметр $\varepsilon=\Delta / E_{0}$, в первом порядке по нему

$$
\begin{aligned}
\bar{c}=\frac{V^{2} d^{2}}{E_{0}}\left[\frac{1}{4 \delta}\left\{\frac{3 \pi}{2} \sqrt{\frac{E_{0}}{\Delta}}-4-\left(\frac{3 \pi}{2} \sqrt{\frac{E_{0}}{\Delta}}-8+8 \varepsilon\right) \gamma^{2}\right\}+\right. \\
\left.+6\left(1-\frac{\pi}{2} \sqrt{\varepsilon}+\varepsilon\right)\right] .
\end{aligned}
$$

Для вычисления параметра Гинзбурга-Леванюка следует еще найти коэффициент при $y^{4}$ в разложении свободной энергии. Основываясь на общем виде $Ф(y)$ из $\left[{ }^{17}\right]$, находим

$b=\frac{1}{N_{0}^{2} v}\left\{\frac{B\{0\}}{12}+\frac{3 V^{4}}{2 \Delta E_{\mathrm{c}}}\left(\frac{1}{\sqrt{E_{0 \Lambda} \Lambda}} \arctan \sqrt{\frac{E_{0}}{\Delta}}+\frac{E_{0}-\Delta}{\left(E_{0}+\Delta\right)^{2}}\right)\right\}$.

В нулевом порядке по $\varepsilon$ второй член в (22) сводится к виду

$$
b^{\prime}=\frac{1}{N_{0}^{2} v} \frac{3 \pi V^{4}}{4 \Delta E_{0}^{2}} \sqrt{\frac{E_{0}}{\Delta}} .
$$

Как видно из (20), константа корреляционной энергии $\bar{c}$ пропорциональна квадрату энергии ведущего фазовый переход вибронного взаимодействия $(V d)$, определяя тем самым и непосредственно масштаб корреляционной длины. Считая $b \approx b^{\prime}$ для узкощельной системы, находим $(\gamma=0)$ 


$$
G_{i} \simeq 11,1 \frac{V^{2} k_{B} T_{C}}{2 \bar{a} \Delta^{3}}\left\{\frac{1}{4 \delta}\left(\frac{3 \pi}{2} \sqrt{\frac{E_{0}}{\Delta}}-4\right)+6\left(1-\frac{\pi}{2} \sqrt{\frac{E_{0}}{\Delta}}\right)\right\}^{-3},
$$

г. е. в этом случае и $G_{i} \sim V^{2}$, в то время как для широкощельной системы $G_{i} \sim B^{2}$.

Проведем теперь численные оценки, положив $\gamma=0$. Для $\mathrm{BaTiO}_{3}$, согласно $\left[{ }^{15,4}\right], \Delta=3$ э $\mathrm{B}, E_{0}=3,7$ эВ $, 2 \bar{a}=1,2 \AA^{-2}, V=1,2$ эВ $\AA^{-1}$, $d=4 \AA, N_{0}^{2} v b=-0,02$ эВ $\AA^{-4}, T_{C}=393, \mathrm{~K}$, эффективный заряд, связанный с мягкой модой $\overline{\mathrm{e}}=2,4$ е. В итоге для коэффициента

$$
\tilde{c}=\frac{\bar{c} v}{\bar{e}^{2}}
$$

корреляционной энергии по спонтанной поляризации получается $\tilde{c}=$ $=7,2 \cdot 10^{-16} \mathrm{~cm}^{2}$, а корреляционная длина $\xi_{0} \sim 15 \AA$. В оригинальной работе Гинзбурга [ $\left.{ }^{1}\right]$ получено $\tilde{c}=3,2 \cdot 10^{-16} \mathrm{~cm}^{2}$, а в $\left[{ }^{21}\right] \tilde{c}=0,5$. $\cdot 10^{-16} \mathrm{~cm}^{2}$. Число Гинзбурга-Леванюка для $\mathrm{BaTiO}_{3} G_{i} \sim 1 \cdot 10^{-4}$, чему соответствует критическая область шириной в несколько сотых градуса. Таким образом, вибронная теория дает примерно такую же оценку характеристик классического сегнетоэлектрика $\mathrm{BaTiO}_{3}$, связанных с пространственными неоднородными флуктуациями параметра порядка, что и рассмотрение на основании эффективных дипольных сил.

Для вибронного сегнетоэлектрика-полупроводника типа $\mathrm{A}_{\mathrm{IV}} \mathrm{B}_{\mathrm{VI}}$, согласно $\left[{ }^{12,13,22-24}\right]$, можно взять $\Delta=0,2$ эВ, $E_{0}=10$ эВ, $d=6,4 \AA$, $2 \bar{a} \sim 0,25 \AA^{-2}, V=0,2$ эВ $\AA^{-1}, T_{C}=100 \mathrm{~K}$, что дает с использованием (21), $(24) \quad \xi \sim 20 \AA, G_{i} \sim 10^{-2}$ и критическую область шириной в градус. При учете динамической части ведущего электрон-фононного взаимодействия в коэффициенте $\bar{c}$ появляется дополнительная температурная зависимость.

В заключение укажем, что вследствие отмеченных в работе приближений настоящие численные результаты следует рассматривать в основном как оценки по порядку величины,

\section{ЛИ Т Е Р А Т У Р A}

1. Гинзбуре В. Л. Физ. твердого тела, 2, вып. 9, 2031-2043 (1960).

2. Леванюк А. П. Ж. экспер. и теор. физики, 36, вып. 3, 810-818 (1959).

3. Гинзбург В. Л., Леванюк А. П., Собянин А. А. Успехи физ. наук, 130, вып. 4, $615-673(1980)$.

4. Kristoffel, N., Konsin, P. Ferroelectrics, 6, № 1, 3-12 (1973); 21, № 4, 477-479 (1978).

5. Кристофель Н. Н., Консин П. Н. Успехи физ. наук, 120, вып. 3, $507-510$ (1976).

6. Берсукер Н. Б., Полингер В. 3. Вибронные взаимодействия в молекулах и кристаллах. М., «Наука», 1983.

7. Бурсиан Э. В., Гиршберг Я. Г., Трунов Н. Н. Изв. вузов. Физика, 24, № 8, 94109 (1981).

8. Kristoffel, N. Czech. J. Phys. B, 34, № 11, 1253-1263 (1984).

9. Волков Б. А., Кушнир В. П., Панкратов О. А. Физ. твердого тела, 24, вып. 2. 415422 (1982).

10. Кочелап В. А., Соколов В. Н., Венгалис Б. Ю. Фазовые переходы в полупроводниках с деформащионным электрон-фононным взаимодействием. Қиев, «Наукова думка», 1984.

11. Гиршберг Я. Г., Тамарченко В. Н. Физ. твердого тела, 18, вып. 4, $1066-1072$ (1976); 18, вып. 11, 3340-3347 (1976).

12. Консин П. Н. Физ. твердого тела, 24, вып. 5, $1321-1327$ (1982),

13. Konsin, P. Ferroelectrics, 45, № 1, 45-50 (1982).

14, Kristoffel, N., Konsin, P. Phys. status solidi, 28, № 2, 731-739 (1968). 
15. Кристофель Н. Н., Консин П. Н. Физ. твердого тела, 13, вып. 9, 2513-2521 (1971).

16. Mailjan, G., Plakida, N. Phys. status solidi (b), 80, № 2, 543-547 (1977).

17. Konsin, $P$. Phys. status solidi (b), 86, № 1, 57-66 (1978).

18. Ландау Л. Д., Лифици Е. М. Статистическая физика, ч. 1. М., «Наука», 1976.

19. I patova, I. P., Maradudin, A. A., Wallis, R. F. Phys. Rev., 155, № 3, 882-895 (1967):

20. Кристофель Н., Консин П. Изв. АН ЭССР. Физ. Матем., 25, № 1, 23-28 (1976).

21. Хотченков А. Г. Изв. вузов. Физика, 23, № 12, 79-80 (1980).

22. Лехцер 3. М., Бурсиан Э. В. В кн.: Сегнетоэлектрики. Л., ЛГПИ, 1979, 89-110.

23. Kawamura, H., Katayama, S., Takano, S., Hotta, S. Solid State Commun., 14, № 3, 259-261 (1974).

24. Sugai, S., Murase, K., Katayama, S., Takaoka, S., Nishi, S., Kawalura, H. Solid State Commun., 24, № 5, 407-409 (1977).

\author{
Ннститут физики \\ Академии наук Эстонской ССР
}

\section{Поступила в редакцию} $16 / \mathrm{V} 1985$

\title{
N. KRISTOFFEL, A. PISTSEV
}

\section{KORRELATSIOONPIKKUSE HINNANG VIBROONSES SENJETTELEKTRIKUS}

On vaadeldud vibroonse senjettelektriku omadusi, mis on seotud fonoonse korrastuse parameetri staatiliste mittehomogeensustega. Hinnatakse korrelatsioonenergia kordajat, korrelatsioonpikkust (osutub võrdeliseks faasisiiret juhtiva tsoonidevahelise vibroonse interaktsiooni energia ruuduga) ning Ginzburg-Levanjuki arvu $\mathrm{BaTiO}_{3}$ ja kitsapiluliste

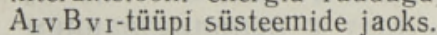

\section{N. KRISTOFFEL, A. PISHCHEV}

\section{CORRELATION LENGTH ESTIMATE IN A VIBRONIC FERROELECTRIC}

The properties of a vibronic ferroelectric connected with the static inhomogeneities of the phonon order parameter are considered. The correlation energy coefficient, the correlation length (appears to be proportional to the squared energy of the interband vibronic interaction driving the phase transition) and the Ginzburg-Levanyuk number for the $\mathrm{BaTiO}_{3}$ and narrow-gap systems of the $\mathrm{A}_{I \mathrm{v}} \mathrm{Bv}_{\mathrm{I}}$ type, are estimated. 